Y. Kitaoka

Nagoya Math. J.

Vol. 69 (1978), 117-120

\title{
REPRESENTATIONS OF QUADRATIC FORMS
}

\author{
YOSHIYUKI KITAOKA
}

0. We have shown in [1]

THEOREM A. Let $L$ be a lattice in a regular quadratic space $U$ over $\boldsymbol{Q}$; then $L$ has a submodule $M$ satisfying the following conditions 1$), 2)$ :

1) $d M \neq 0, \operatorname{rank} M=\operatorname{rank} L-1$, and $M$ is a direct summand of $L$ as a module.

2) Let $L^{\prime}$ be a lattice in some regular quadratic space $U^{\prime}$ over $\boldsymbol{Q}$ satisfying $d L^{\prime}=d L$, rank $L^{\prime}=\operatorname{rank} L, t_{p}\left(L^{\prime}\right) \geq t_{p}(L)$ for any prime $p$. If there is an isometry $\alpha$ from $M$ into $L^{\prime}$ such that $\alpha(M)$ is a direct summand of $L^{\prime}$ as a module, then $L^{\prime}$ is isometric to $L$.

Our aim is to remove such a restriction in 2) that $\alpha(M)$ is a direct summand of $L^{\prime}$ as a module:

THEOREM B. Let $L$ be a lattice in a regular quadratic space $U$ over $\boldsymbol{Q}$; then $L$ has a submodule $M$ with $\operatorname{rank} M=\operatorname{rank} L-1, d M \neq 0$ which is a direct summand of $L$ as a module and satisfies

$\left.{ }^{*}\right)$ let $L^{\prime}$ be a lattice in some regular quadratic space $U^{\prime}$ over $\boldsymbol{Q}$ satisfying $d L^{\prime}=d L, \operatorname{rank} L^{\prime}=\operatorname{rank} L, t_{p}\left(L^{\prime}\right) \geq t_{p}(L)$ for any prime $p$; if there is an isometry a from $M$ into $L^{\prime}$, then $L^{\prime}$ is isometric to $L$.

\section{Notations and some lemmas}

We denote by $\boldsymbol{Q}, \boldsymbol{Z}, \boldsymbol{Q}_{p}$ and $\boldsymbol{Z}_{p}$ the rational number field, the ring of rational integers, the $p$-adic completion of $\boldsymbol{Q}$, and the $p$-adic completion of $Z$, respectively. For a quadratic space $U$ we denote $Q(x), B(x, y)$ the quadratic form and the bilinear form associated with $U(2 B(x, y)$ $=Q(x+y)-Q(x)-Q(y))$, and for a lattice $L$ in $U d L$ stands for the discriminant of $L$. For two ordered sets $\left(a_{1}, a_{2}, \cdots, a_{n}\right),\left(b_{1}, b_{2}, \cdots, b_{n}\right)$, we define the order $\left(a_{1}, a_{2}, \cdots, a_{n}\right) \leq\left(b_{1}, b_{2}, \cdots, b_{n}\right)$ by either $a_{i}=b_{i}$ for $i<k$ and $a_{k}<b_{k}$ for some $k \leq n$ or $a_{i}=b_{i}$ for any $i$.

Received February 18, 1977. 
Let $L$ be a lattice in a regular quadratic space over $\boldsymbol{Q}_{p}$; then $L$ has a Jordan splitting $L=L_{1} \perp L_{2} \perp \cdots \perp L_{k}$, where $L_{i}$ is a $p^{a i}$-modular lattice and $a_{1}<a_{2}<\ldots<a_{k}$. We denote by $t_{p}(L)$ the ordered set $\underbrace{\left(a_{1}, \cdots a_{1}\right.}_{\text {rank } L_{1}}$, $\cdots, \underbrace{a_{k}, \cdots, a_{k}}_{\text {rank } L_{k}})$. For a lattice $L$ in a regular quadratic space over $\boldsymbol{Q}$ we abbreviate $t_{p}\left(Z_{p} L\right)$ to $t_{p}(L)$.

LEMMA 1. Let $L$ be a lattice in a regular quadratic space $U$ over $\boldsymbol{Q}_{p}$; then $L$ has a submodule $M$ satisfying the following conditions:

1) $d M \neq 0, \operatorname{rank} M=\operatorname{rank} L-1$, and $M$ is a direct summand of $L$ as a module.

2) Let $L^{\prime}$ be a lattice in $U$ containing $M$; then $L^{\prime}=L$ if $d L^{\prime}=d L$ and $t_{p}\left(L^{\prime}\right) \geq t_{p}(L)$.

This was proven in [1], and we called $M$ a characteristic submodule of $L$.

LEMMA 2. Let $L$ be a lattice with the scale $\subset Z$ in a regular quadratic space $U$ over $\boldsymbol{Q}$ with $\operatorname{dim} U \geq 3$. If a direct summand $M$ of $L$ satisfies

1) $M_{p}$ is a characteristic submodule of $L_{p}$ if $p \mid 2 d L$,

2) $d M=q^{r} m$ where $q$ is a prime with $q \nmid 2 d L$ and $r \geq 0$, and $p \mid 2 d L$ if $p \mid m$,

then $M$ satisfies the conditions 1$), 2)$ in Theorem $A$.

This is a remark in $\S 1$ in [1].

LEMMA 3. Let $L$ be a lattice in a regular quadratic space $U$ over $\boldsymbol{Q}$ with $\operatorname{dim} U>2$, and let $S$ be a finite set of finite primes such that $2 \in S$, and $L_{p}$ is unimodular for $p \notin S$. For a given $u_{p} \in L_{p}(p \in S)$ there is a prime $q \notin S$ and $a$ vector $u \in L$ such that $u$ and $u_{p}$ are sufficiently near for $p \in S$, and $Q(u) \in \boldsymbol{Z}_{p}^{\times}$for $p \neq q, p \notin S$, and $Q(u) \in q \boldsymbol{Z}_{q}^{\times}$.

Proof. We can take a vector $v_{1}$ in $L$ such that $v_{1}$ is sufficiently near to $u_{p}$ for $p \in S$ and $Q\left(v_{1}\right) \neq 0$, and put $T=\left\{p ; p \notin S, Q\left(v_{1}\right) \notin \boldsymbol{Z}_{p}^{\times}\right\}$. Then there is a vector $v_{2} \in L$ such that $Q\left(v_{2}\right) \in Z_{p}^{\times}$for $p \in T$ and $\pm d Z\left[v_{1}, v_{2}\right]$ is not in $\boldsymbol{Q}^{\times 2}$ since $L_{p}$ is unimodular for $p \notin S$. Put $\tilde{L}=Z\left[v_{1}, v_{2}\right] \subset L$, and take a vector $v$ in $\tilde{L}$ such that $v$ and $v_{1}$ (resp. $v_{2}$ ) are sufficiently near for $p \in S$ (resp. $p \in T$ ). There is a basis $\left\{e_{1}, e_{2}\right\}$ of $\tilde{L}$ such that $\left(B\left(e_{i}, e_{j}\right)\right)=d\left(\begin{array}{cc}a & b / 2 \\ b / 2 & c\end{array}\right)$ where $a, b, c \in \boldsymbol{Z}, d \in \boldsymbol{Q}^{\times}$, and $(a, b, c)=1$. Since 
$Q\left(\tilde{L}_{p}\right) \cap \boldsymbol{Z}_{p}^{\times} \neq \phi$ for $p \notin S$, a prime $p$ with $d \notin Z_{p}^{\times}$is contained in $S$. Noting $Q(v) \in Z_{p}^{\times}$for $p \in T$, we have only to prove Lemma in case that $L \cong\left(\begin{array}{cc}a & b / 2 \\ b / 2 & c\end{array}\right)$, by scaling of $1 / d$, and $u_{p}=v$ for $p \in S \cup T$. Thus we may assume that $L=Z\left[e_{1}, e_{2}\right],\left(B\left(e_{i}, e_{j}\right)\right)=\left(\begin{array}{cc}a & b / 2 \\ b / 2 & c\end{array}\right)(a, b, c)=1, D=b^{2}$ $-4 a c$ is not a square in $\boldsymbol{Q}$, and $p \nmid D$ if $p \notin S$. Moreover $v \in L$ is given. By a classical theory we may suppose that $a$ is a prime number $\notin S$ by scaling of \pm 1 if necessary. Put $k=Q(\sqrt{D})$ and $\tilde{A}=Z[a,(b+\sqrt{D}) / 2]$, $A=(a,(b+\sqrt{D}) / 2)$ ( $=$ the ideal generated by $a$ and $(b+\sqrt{D}) / 2)$; then the norm of $A$ is $a$ and for $\alpha=a x+(b+\sqrt{D}) y / 2(x, y \in \boldsymbol{Q}), N(\alpha)=a\left(a x^{2}\right.$ $\left.+b x y+c y^{2}\right)$. Hence $Q\left(x e_{1}+y e_{2}\right)=N(\alpha) / a$. Thus we may consider $\tilde{A}$, $N(\alpha) / a$ as $L, Q(\alpha)$ respectively, and are given an element $v$ in $\tilde{A}$. Put $J=\left(\prod_{p \in S} p\right)^{t}$; then to complete the proof we need only show that there is an element $u$ in $\tilde{A}$ and a prime number $q \notin S$ such that $u \equiv v \bmod J$, and $Q(u) \in Z_{p}^{\times}$for any prime $p \notin S, p \neq q$, and $Q(u) \in q Z_{q}^{\times}$. Put $(v)=B C$ where $B, C$ are integral ideals and for a prime ideal $E|J, E|(v)$ if and only if $E \mid B$. Hence $(J, C)=1$. Take a prime ideal $I$ with a prime norm $q \notin S$ such that $I=\tilde{u} C A^{-1}, \tilde{u} \equiv 1 \bmod ^{\times} J$. Put $u=\tilde{u} v$; then $(u)=I A B$ $\subset A$. Hence $u \in A$, and $u \equiv v \bmod J$. Moreover $Q(u)=N(u) / a= \pm N I$. $N B$, where $N I=q$ is a prime $\notin S$ and $N B \in Z_{p}^{\times}(p \notin S)$. We must show $u \in \tilde{A}$. Put $D=f^{2} d$ where $d$ is the discriminant of $Q(\sqrt{ } \bar{D})$; Since $p \mid J$ for $p \mid f, u-v=(\tilde{u}-1) v \in f A . \quad v \in \tilde{A}$ and $N A \nmid f$ imply $u \in \tilde{A}$. This completes a proof.

\section{Proof of Theorem B}

Without loss of generality we may assume that the scale of $L$ is contained in $Z$. If $\operatorname{rank} L=2$, then the proof of Theorem $\mathrm{A}$ in [1] shows that Theorem $\mathrm{B}$ is true. Assume $\operatorname{rank} L \geq 3$. Then take an element $u_{p}$ in $L_{p}$ for $p \mid 2 d L$ such that $u_{p}^{\perp}$ is a characteristic submodule of $L_{p}$. From Lemma 3 follows that there is an element $u$ in $L$ and a prime $q \nmid 2 d L$ such that $u$ and $u_{p}$ are sufficiently near in $L_{p}$ for $p \mid 2 d L$ and $Q(u) \in Z_{p}^{\times}$for $p \notin S, p \neq q$, and $Q(u) \in q Z_{q}^{\times}$. Since $u$ and $u_{p}$ are sufficiently near, there is a unit $\varepsilon_{p} \in Z_{p}$ such that $Q(u)=\varepsilon_{p}^{2} Q\left(u_{p}\right)$. Hence there is an isometry $\beta_{p} \in O\left(L_{p}\right)$ such that $\beta_{p}(u)=\varepsilon_{p} u_{p}$. Put $M=u^{\perp}$ in $L$; then $M_{p}$ is a characteristic submodule of $L_{p}(p \mid 2 d L)$, and $d M_{q} \in q Z_{q}^{\times}$, and $d M_{p} \in Z_{p}^{\times}$for $p \notin S, p \neq q$. Therefore $M$ satisfies the conditions 1 ), 
2) in Theorem A by Lemma 2. Thus we have only to prove that $\alpha(M)$ is a direct summand of $L^{\prime}$ for an isometry $\alpha$ from $M$ into a lattice $L^{\prime}$ in 2) in Theorem B. Extend $\alpha$ to an isometry from $U$ to $U^{\prime}$, and put $L^{\prime \prime}$ $=\alpha^{-1}\left(L^{\prime}\right)$. Since $M_{p}$ is a characteristic submodule of $L_{p}, L_{p}^{\prime \prime}=L_{p}$ for $p \mid 2 d L$. If $p \nmid 2 d L, L_{q}^{\prime \prime}$ is unimodular. Hence $M_{p}$ is a direct summand of $L_{p}^{\prime \prime}$ since $d M_{p} \in \boldsymbol{Z}_{p}^{\times}$or $p \boldsymbol{Z}_{p}^{\times}$. Therefore $M$ is a direct summand of $\alpha^{-1}\left(L^{\prime}\right)$ $=L^{\prime \prime}$. This completes a proof of Theorem $\mathrm{B}$.

\section{REFERENCES}

[1] Y. Kitaoka, Representations of quadratic forms and their application to Selberg's zeta functions, Nagoya Math. J. vol. 63 (1976), 153-162.

[ 2 ] O. T. O'Meara, Introduction to quadratic forms, Springer-Verlag, 1963.

Department of Mathematics

Nagoya University 